\title{
Mise au point de chambres de refroidissement pour l'étude de la gélivité des bourgeons. Application au cas de la vigne
}

\author{
D Flura 1, B Itier 1, O Brun 2, B Durand 1, S Masson 1 \\ 1 INRA, station de bioclimatologie, 78850 Thiverval-Grignon; \\ 2 GCEV MUMM vignobles et recherches, 11, avenue de Champagne, 51200 Epernay, France
}

(Reçu le 10 janvier 1991; accepté le 10 mars 1991)

\begin{abstract}
Résumé - Les auteurs décrivent 2 chambres de refroidissement utilisées pour les études de gélivité des bourgeons in situ. La première est une chambre de refroidissement convectif élaborée à partir d'éléments de congélateurs. La deuxième cherche à reproduire le phénomène de refroidissement radiatif qui a lieu au cours des nuits de gelées de printemps afin de permettre les dépôts de rosée et de givre qui se produisent dans les conditions naturelies.
\end{abstract}

chambre / refroidissement / gélivité

Summary - Two types of cooling chambers designed for studying bud sensitivity to frost. The authors describe 2 cooling chambers used to study frost sensitivity of vine buds. The first is a classical convective cooling system which can be run in the field (fig 1). The second is designed to provide buds with the radiative cooling which occurs during spring frosts. This chamber reproduces part of the system designed by Went (1976), Gill and Waister (1976) and Marcellos (1981). Figure 3 reproduces the schema of the chamber. Radiative cooling is provided by means of evaporating nitrogen at roof level in the upper part (3). Warm air is circulated in the middle part in order to maintain the polyethylene walls (4) free of water and transparent to thermal infrared radiation. This radiative cooling system makes dew or rime deposition possible, as in natural conditions. This is illustrated in figure 4 where bud temperature (---- and - - -) is kept lower than both air temperature (-) and dew point (-๑) during cooling.

chamber / cooling / frost sensitivity

\section{INTRODUCTION}

L'étude de la gélivité des bourgeons, c'est-à-dire de leur sensibilité au gel, s'est heurtée à la difficulté que représente la rareté du phénomène de gel dans les conditions naturelles. Afin d'augmenter le nombre d'observations susceptibles de fournir une relation entre la gélivité et des facteurs déterminants tels que la phénologie, le mouillage des bourgeons et/ou la teneur en bactéries glaçogènes (Itier et al, 1991) nous avons été conduits à développer des chambres de refroidissement. L'objectif à atteindre consiste à élaborer des instruments permettant de reproduire des types de refroidissement les plus proches de ceux rencontrés dans les conditions naturelles, afin de les utiliser pour l'étude du déterminisme des températures de rupture de surfusion, synonyme de la mort par le gel.
Pour y parvenir nous avons, tout d'abord, élaboré une chambre à refroidissement convectif, susceptible d'être mise en œuvre directement sur le terrain autour d'un cep de vigne en place. Compte tenu de l'impossibilité d'obtenir avec ce type de matériel une condensation ou un givrage des bourgeons, nous avons été conduits à mettre au point dans un deuxième temps une chambre de refroidissement fonctionnant à partir d'un puits radiatif artificiel.

\section{CHAMBRE À REFROIDISSEMENT CONVECTIF IN SITU}

La chambre (fig 1) est constituée de 3 éléments : 2 demi-congélateurs (1) comprenant chacun une plaque de refroidissement (3) et pouvant s'accoler l'un à l'autre après avoir inséré un cep de 

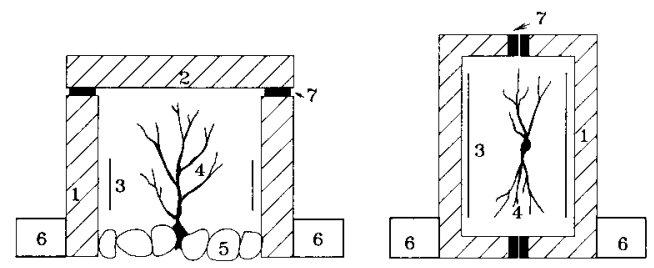

Fig 1. Schéma de la chambre à refroidissement convectif. a) vue en coupe transversale; b) vue du dessus, couvercle ouvert. (1) structure isolante, (2) couvercle, (3) plaque froide, (4) pied de vigne, (5) sacs remplis de billes de polystyrène, (6) compresseur, (7) joint.

vigne (4) et un couvercle (2) assurant l'étanchéité vers le haut. Une fois le système en place, on assure l'étanchéité vers le bas, afin de prévenir l'écoulement de l'air froid créé dans l'enceinte, au moyen de sacs remplis de billes de polystyrène (5). Une régulation de la descente en température conduite à partir d'un système de mise en route/arrêt des groupes froid conduirait à de trop grands à-coups dans la variation de la température. Aussi, avons-nous opté pour un fonctionnement continu des groupes froid associé à un fonctionnement discontinu de résistances chauffantes. La mise en route des résistances est pilotée par une centrale d'acquisitions sur laquelle nous avons programmé une décroissance de température voisine de $2{ }^{\circ} \mathrm{C}$ par $\mathrm{h}$. Dès que la température enregistrée dans la chambre s'écarte de plus de $0,5^{\circ} \mathrm{C}$ de la température prévue, la coupure des résistances s'effectue.

À l'intérieur de la chambre de refroidissement, 10 bourgeons sont sélectionnés sur le pied de vigne pour être piqués par des thermocouples en cuivre constantan, et l'on mesure directement l'écart entre la température du bourgeon et celle de l'air, repérée par un thermocouple piqué dans un morceau de bois mort. L'augmentation brusque de cet écart est la traduction de la rupture de surfusion au sein du bourgeon. Un exemple d'enregistrement est donné sur la figure 2 , où sont portés en fonction du temps, d'une part, l'évolution de la température d'un morceau de bois mort, utilisé en tant qu'indice actinothermique, et d'autre part, l'écart de température entre différents bourgeons et cet indice. On observe bien les accidents que sont les exothermes résultant de la rupture de surfusion.

La chambre de refroidissement a été utilisée au cours des printemps 1987, 1988 et 1989 sur le vignoble Mumm à Avize en Champagne (Itier et al, 1991). Elle a permis d'établir des relations entre la température de surfusion des bourgeons de vigne (Vitis vinifera L Chardonnay) et des variables telles que la phénologie des bourgeons, la teneur en bactéries glaçogènes et le mouillage de la surface des bourgeons provoqué de façon artificielle. En effet, ce type de chambre ne permet pas d'obtenir un dépôt de rosée ou de givre sur les bourgeons. La température des bourgeons est toujours légèrement supérieure à celle de l'air puisqu'il s'agit d'un refroidissement convectif. Ainsi, lorsque la température de l'air atteint le point de rosée, le dépôt d'eau, ou de givre, s'effectue sur les plaques de refroidissement. Contrairement à ce qui se passe dans les conditions naturelles, on est toujours placé dans des conditions d'air sous-saturé. Le dépôt de rosée ou de givre pouvant avoir une incidence sur la sensibilité des bourgeons au gel, il était donc indispensable de construire un système qui permette à ce phénomène de se réaliser. C'est à cet objectif que répond la construction d'une chambre à refroidissement radiatif.

\section{CHAMBRE À REFROIDISSEMENT RADIATIF}

Afin d'obtenir un dépôt de rosée ou de givre, il faut que la température des bourgeons soit inférieure à la température de l'air, comme c'est le cas lors des nuits de gel. Pour y parvenir, il faut produire un déficit du rayonnement au niveau du bourgeon. II s'agit donc de mettre en œuvre une chambre comportant un puits radiatif. Ce type d'appareil n'existant pas dans le commerce, nous avons décidé d'entreprendre nous-mêmes cette réalisation.

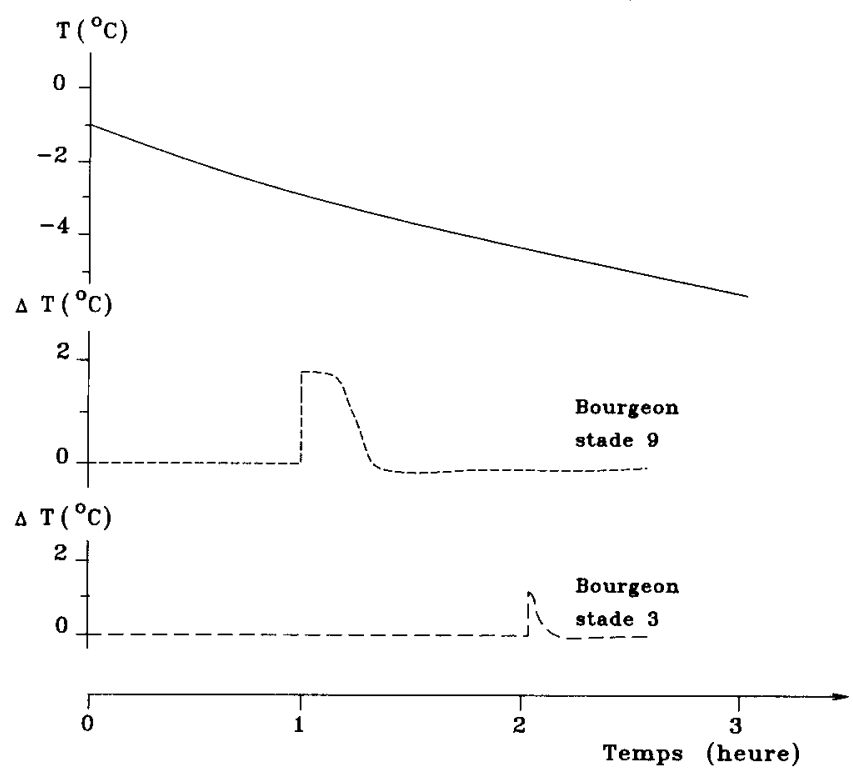

Fig 2. Exemple d'exothermes indiquant la température de rupture de surfusion. indice actinothermique : -; écart température bourgeons et indice actino-thermique :---- et ---. 
La chambre s'apparente à celle réalisée par Went (1976). Elle combine le mode de production du puits radiatif décrit dans la chambre de Gill et Waister (1976) avec les précautions prises par Marcellos (1981) pour éviter les mouvements de convection interne dans la partie où se trouve le végétal. Elle comporte 3 parties (cf fig 3 ).

La cellule supérieure (3) dévolue au refroidissement radiatif comporte une paroi supérieure à laquelle est soudée un serpentin dans lequel on fait circuler de l'azote liquide en cours d'évaporation. Cet azote provient d'un réservoir maintenu sous pression, la pression ayant été ajustée de telle sorte que la vitesse de refroidissement des bourgeons soit compatible avec les refroidissements naturels $(0,6$ bar pour un refroidissement de $2{ }^{\circ} \mathrm{C} / \mathrm{h}$ ).

La cellule intermédiaire (2) est, comme dans le cas de la chambre de Marcellos (1981), bordée par 2 parois de polyéthylène entre lesquelles circule un air chauffé par des résistances et desséché par le silicagel. Ceci permet d'éviter que le dépôt de buée et de givre sur les parois ne vienne éteindre la fonction puits radiatif recherchée. En effet, la transparence du polyéthylène aux infrarouges thermiques est liée à l'absence de dépôt surfacique. Par ailleurs, le fait que la paroi inférieure du compartiment intermédiaire soit chaude évite les mouvements de convection dans la chambre inférieure. Ces mouvements sont à proscrire si l'on veut reproduire les conditions de stabilité thermique régnant pendant les nuits de refroidissement radiatif.

La cellule inférieure (1) dont les parois sont recouvertes de papier argenté, contient des thermocouples permettant de repérer les exothermes résultant de la rupture de surfusion.

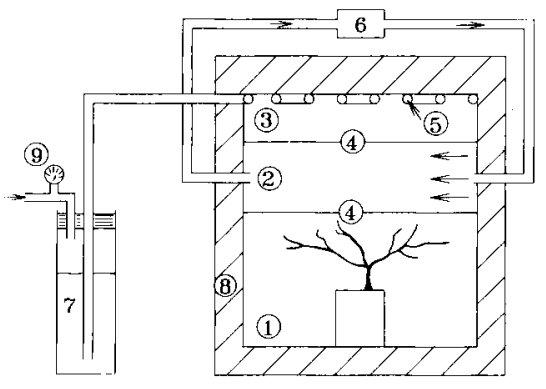

Fig 3. Schéma de la chambre à refroidissement radiatif : (1) compartiment inférieur comprenant les sarments et le dispositif de mesure, (2) compartiment intermédiaire parcouru par de l'air sec et chaud, (3) compartiment supérieur, (4) parois de polyéthylène, (5) serpentin dans lequel circule lazote liquide, (6) éléments de chauffage et de dessication, (7) réservoirs d'azote liquide sous pression, (8) bâti isolant de la chambre, (9) manomètre.

\section{RÉSULTATS}

La figure 4 donne un exemple de décroissance de températures obtenues dans la chambre ainsi que d'écarts entre température des bourgeons et température de l'air. La mesure de la température de l'air a été réalisée au moyen d'un thermocouple très fin $(\varnothing \approx 20 \mu \mathrm{m})$ abrité du déficit radiatif du plafond par une plaque d'aluminium recouverte d'un isolant. Les températures de bourgeons sont toujours inférieures à la température de l'air, ce qui correspond bien à un des objectifs affichés. Par ailleurs, les températures des bourgeons sont inférieures à la température du point de rosée mesurée au moyen d'un hygromètre à effet Peltier dès que celle-ci est rejointe par la température de l'air. La figure 4 montre en effet que tant que l'arrêt du refroidissement n'a pas lieu (avant $17 \mathrm{~h} 20$ en abscisse) il y a égalité entre $T_{a}$ et $T_{r}$, et donc probablement dépôt de rosée sur les bourgeons plus froids. Nous avons d'ailleurs pu constater, lors d'arrêt de l'expérience, ce mouillage par la rosée à la surface des bourgeons.

La chambre de type radiatif réalisée a été utilisée en 1989 sur le vignoble Mumm en Champagne. Afin de pouvoir valider les résultats obtenus au moyen de cette chambre, nous avons procédé en 2 étapes :

- nous avons tout d'abord vérifié, à l'intérieur de la chambre convective, que les températures moyennes de rupture de surfusion à un stade donné étaient les mêmes, que l'on opère sur un pied de vigne en place ou sur des sarments prélevés;

- nous avons ensuite vérifié que la moyenne des températures de rupture de surfusion des bourgeons était la même, que l'on opère à partir

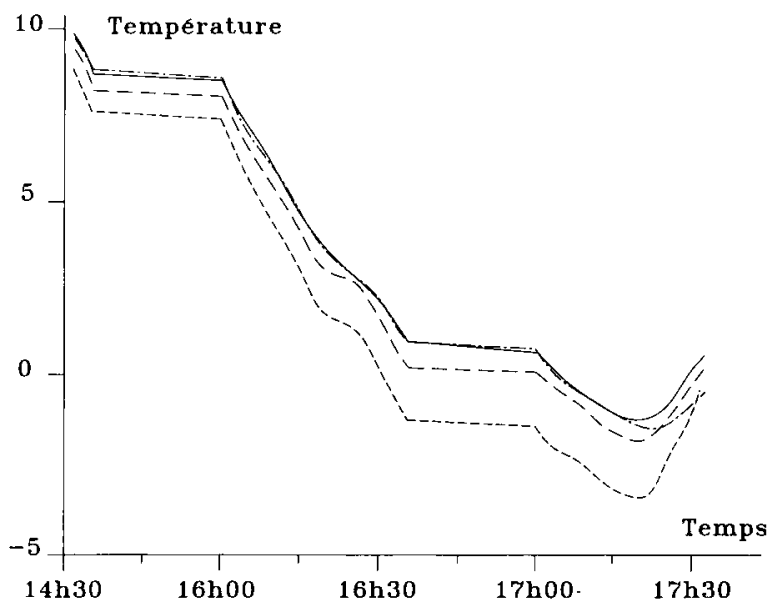

Fig 4. Exemple d'évolution simultanée de différentes températures enregistrées dans la chambre à refroidissement radiatif. Température de l'air : -; température des bourgeons : - - et - . -; température du point de rosée : $\rightarrow$. 
de la chambre à refroidissement convectif ou à partir de la chambre à refroidissement radiatif, pour autant que l'on reste au-dessus du point de rosée.

\section{CONCLUSION}

Les 2 appareils réalisés ont permis d'effectuer un suivi de la gélivité des bourgeons tout au long d'une saison de végétation. Les outils mis au point devraient permettre dans le futur d'étendre les études réalisées sur vigne aux arbres fruitiers, que ce soit en emprisonnant une branche sur pied à l'intérieur de la chambre convective ou, plus simplement, en travaillant sur des organes végétaux appartenant à des rameaux prélevés. Par ailleurs, la chambre radiative devrait pouvoir être utilisée dans le cadre des études conduites avec les pathologistes pour caractéri- ser l'influence de la durée de mouillage sur le développement des maladies cryptogamiques.

\section{RÉFÉRENCES}

Gill PA, Waister PD (1976) Design and performance of a portable frost chamber for the investigation of hardiness of strawberries. J Hortic Sci 51, 509-513

Itier B, Flura D, Brun O, Luisetti J, Gaignard JL, Choisy C, Lemoine $G$ (1991) Analyse de la gélivité des bourgeons de vigne (expérimentation in situ sur le vignoble champenois). Agronomie 11, 169-174

Marcellos $H$ (1981) A plant freezing chamber with radiative and convective energy exchange. $J$ Agric Eng Res 26, 403-408

Went FW (1976) Le phytotron comme trait d'union entre la conception et la réalité d'une plante. In: Etudes de biologie végétale. Hommage au Professeur Pierre Chouard ( $R$ Jacques, ed), R Jacques, Paris, 1976, 321-330 